\title{
PROBLEMAS RELATIVOS A LA PREVISIÓN DE LAS NECESIDADES DE MANO DE OBRA EN AMÉRICA LATINA *
}

\author{
VÍCTOR L. URQUIDI \\ El Colegio de México
}

I. EN LOS ÚlTIMOS AÑos se viene prestando creciente atención en América Latina a diversos problemas que afectan la capacidad productiva futura de la región. El énfasis inicial -por ejemplo, en los estudios de la Comisión Económica para América Latina- puesto en las tendencias, la estructura y las perspectivas de la demanda fue de gran utilidad para empezar a adoptar grandes lineamientos de política económica, en particular para derivar del lento crecimiento previsible de la demanda externa (y más precisamente del de la capacidad de pago generada por las exportaciones) algunas conclusiones de carácter fundamental sobre el papel que debe desempeñar una demanda interna autónoma como motor del desarrollo económico, a través del gasto público, la industrialización y el mejoramiento de la distribución del ingreso. Aceptada gradualmente en América Latina una nueva "estrategia de la demanda", se empezaron a encaminar los esfuerzos de los investigadores a descubrir y valorar los factores favorables o desfavorables al cambio en la estructura de la producción. Esto condujo a iniciar una serie de estudios, en los propios países lo mismo que en los diversos organismos internacionales, en materia de evolución demográfica, evaluación de recursos naturales, estructura e instituciones agrarias, estado de la técnica y capacidad para absorber nueva tecnología, estructura educativa, naturaleza y utilidad de la infraestructura económica existente, calidad y productividad del capital industrial, nivel de capacitación de la mano de obra en general y de los cuadros técnicos medios y superiores en especial, administración pública y reformas institucionales necesarias para acelerar el desarrollo, etc. Dentro de este conjunto, la formación del hombre, no sólo en el aspecto cultural general sino como elemento clave en la producción, tiene importancia primordial. De allí que en la actualidad sea necesario relacionar las aspiraciones económicas de América Latina

* Trabajo presentado al Seminario sobre Previsiones a Largo Plazo de las Necesidades de Mano de Obra y las Políticas de Educación, auspiciado por la Organización de Cooperación y Desarrollo Económicos, París, y el Instituto Nacional de Planificación del Perú, Lima, 22-27 de marzo de 1965. Aquí se publica con sólo ligeras modificaciones y la adición de referencias bibliográficas. 
con la acción educativa y de otra índole tendientes a elevar la productividad y a facilitar la adaptación a nuevos y más complejos niveles de tecnología.

El problema hasta cierto punto paralelo a que se han enfrentado diversos países del sur de Europa, comprendidos en el llamado Proyecto Regional del Mediterráneo (PRM) de la Organización de Cooperación y Desarrollo Económicos, ha sido objeto de mucha mayor atención que en América Latina, y se están obteniendo ya algunos resultados tangibles de su estudio. ${ }^{1}$ Aunque el atraso de los sistemas educativos y de la formación de mano de obra reviste mayor gravedad en América Latina, el haber diferido en parte su consideración permite al menos derivar algún beneficio de la experiencia europea en cuanto a planteamiento, metodología y, a medida que se adopten programas de acción, política a seguir.

II. Pueden señalarse cuatro diferencias cualitativas de base entre la experiencia europea del PRM y la situación latinoamericana:

a) El incremento demográfico latinoamericano es casi el triple del aumento que acusan los países del PRM (salvo Turquía, en que se asemeja). Según las últimas estimaciones, que toman en cuenta importantes correcciones a los últimos censos, la población de América Latina crecerá hasta 1980 , dadas las tendencias actuales, a razón del $2.9 \%$ anual; en un grupo de quince países, incluidos los dos más poblados (Brasil y México), la tasa será no inferior a 3.1\%.2 Tan sólo en México, según nuevas proyecciones, el crecimiento será del $3.6 \%$ anual hacia $1970-1975.3$ Junto con la mayor dinámica demográfica latinoamericana, con todas las consecuencias que tiene en la composición por edades de la fuerza de trabajo y en las relaciones de dependencia, el tamaño de las familias, etc., se debe considerar una tasa de migración entre áreas rurales y urbanas mayor que en Europa y, salvo Ia emigración temporal de trabajadores mexicanos a los Estados Unidos (ya muy limitada), la ausencia de alivio por emigración; antes bien, se manifiestan tipos de emigración de ciertos países latinoamericanos cuyo efecto es negativo y casi irreparable, por la salida de técnicos.

b) La estructura productiva de los países del PRM, según se manifiesta en la distribución de la población activa por sectores de actividad, es más avanzada que la latinoamericana en general. Sólo en uno de los seis países del PRM se manifiesta una estructura acusadamente agraria: Turquía, donde el $75 \%$ de la población activa tiene ocupación en la agricultura y sólo el $10 \%$ en la industria. En el resto, la agricultura absorbe un máximo de $57 \%$ de la población activa (Yugoslavia) y un mínimo de $28 \%$ (Italia), y la industria un mínimo

1 OECD, París, The Mediterranean Regional Project: Herbert S. Parnes, editor, Planning Education for Economic and Social Development, 1965; y Country Reports: Greece; Italy; Portugal; Spain; Turkey; Yugoslavia, informes publicados en 1965.

2. Véase mi ponencia presentada al Congreso Mundial de Población, 1965, “El crecimiento demográfico y el desarrollo económico latinoamericano", reproducida en Demografía y Economía, Vol I, Núm. 1, El Colegio de México, 1967.

3 Raúl Benítez Zenteno y Gustavo Cabrera Acevedo, Proyecciones de la población de México, 1960-1980, México, Banco de México, 1966. 
de $17 \%$ en Yugoslavia y un máximo de $32 \%$ en España. Las condiciones de Yugoslavia se aproximan en general a las latinoamericanas. Aun en el sur de Italia, la absorción de la población activa en la agricultura (el $45 \%$ ) es inferior al promedio latinoamericano. Dada en muchos casos la menor dimensión geográfica de los países del PRM, y las mejores comunicaciones internas, la interacción de los sectores agrícola e industrial es indudablemente mayor en Europa. En América Latina existe una separación más señalada, geográfica y culturalmente, entre la vida rural y la urbana; en Europa no hay tanta disparidad entre ciudades grandes y pequeñas, ni entre éstas y las zonas rurales.

c) Los sistemas educativos de los países del PRM han evolucionado más, en general, que los latinoamericanos. Aun sin contar la obvia tradición cultural y educativa europea, las proporciones de escolaridad de los países del PRM, salvo en parte el caso de Turquía, son indiscutiblemente superiores, respecto a los niveles de educación, a las registradas en la mayor parte de los países latinoamericanos. Son también mayores las proporciones entre educación técnica y educación general. El gasto total en educación, salvo en España, parece ser una proporción más elevada del producto nacional bruto en los países del PRM que en la gran mayoría de los latinoamericanos, donde en muy contados casos excede del 2 por ciento.

d) El proceso de absorción y difusión de la tecnología ha sido en los países del PRM probablemente más generalizado, continuo y directo que en América Latina, en términos generales (salvando casos especiales). Esto, que se relaciona en parte con el sistema educativo y con los medios de comunicación y difusión cultural, tiene también que ver con las formas en que, en América Latina, la tecnología moderna ha llegado, vinculada a las inversiones extranjeras. Con excepción de gran parte de la agricultura, en que ha habido desarrollos tecnológicos autónomos, la tecnología ha sido traída a América Latina por el capital extranjero privado, y sólo en muy pequeña medida se han destinado recursos a la investigación y adaptación tecnológicas con independencia de esa vinculación. Puede afirmarse que en los países del PRM ha habido en su mayoría una iniciativa tecnológica propia que facilita a su vez una industrialización mucho menos dependiente de los problemas de la inversión privada extranjera (en Yugoslavia, por supuesto, con total independencia).

Las anteriores consideraciones son importantes porque tienen consecuencias en el planteamiento del problema en América Latina. No hay duda de que el incremento demográfico tan rápido y la estructura de edades a que da lugar obligan a considerar con mucha más urgencia que en los países del PRM la reforma de los sistemas educativos y la adopción de medidas para capacitar a la mano de obra. Por otro lado, sin embargo, la probable estructura futura de la población económicamente activa en América Latina, al caracterizarse por proporciones mayores ocupadas en la agricultura que en Europa, tal vez imponga al sistema educativo tareas relativamente menos gravosas en la enseñanza media y superior. En cambio, las tasas de incremento anual de la escolaridad tendrán que ser mayores en América Latina, a todos los niveles. El rezago tecnológico requerirá, además, orientar parte importante del flujo escolar superior a las tareas de investigación 
y no sólo a la actividad productiva directa. Los bajos niveles de escolaridad, y las elevadas tasas de deserción a que la estructura del sistema educativo da origen y a que contribuyen las condiciones económicas de los educandos, significan, también, que la tarea de la preparación de la mano de obra latinoamericana no puede recaer exclusivamente en el sistema educativo sino que tendrá que ser asumida simultáneamente por el sector industrial, comercial y de servicios.

III. El examen de estos problemas adquiere urgencia asimismo por otra serie de consideraciones. América Latina ha adoptado como meta un crecimiento económico del producto por habitante de $2.5 \%$ anual, que es un poco superior al registrado en los últimos seis años, cuando la población crecía también menos rápidamente. Cualesquiera que sean los supuestos sobre la inversión necesaria para obtener el aumento deseado del ingreso por habitante, la expansión y el cambio de estructura de la población tenderán a hacer más difícil el logro de las metas de inversión (puesto que la dinámica demográfica favorece el consumo), a la par que se acentuarán el conflicto de prioridad entre inversión directamente productiva e inversión en infraestructura técnica y educativa (inversión social en general). Aun suponiendo que se encuentren criterios para resolver este conflicto y optimizar la inversión en actividades directamente productivas, no existe segux idad alguna de que las tasas de inversión alcanzables, y los incrementos del producto bruto resultantes, sean suficientes para absorber en ocupación adecuadamente remunerada los aumentos de la población en edad de trabajo; es decir, hay dudas respecto a la posibilidad de aumentar la relación entre la fuerza de trabajo y la población total, así como con relación a la de reducir apreciablemente, dentro de la fuerza de trabajo, las proporciones de desocupados y de subocupados. Es de suponer que las tasas de participación en el trabajo de las mujeres en determinados grupos de edades se eleven, y que, a medida que se amplíe el sistema educativo, se reduzcan las de los hombres en edades tempranas; pero ello no garantiza que se reduzca la subocupación, abierta o disfrazada.

La dificultad que existe para relacionar las metas de crecimiento con las proyecciones de la fuerza de trabajo necesita abordarse desde varios puntos de vista. A priori, supone la necesidad de adoptar metas de crecimiento económico más ambiciosas. En los países del PRM, junto con las condiciones demográficas menos dramáticas que las latinoamericanas, se han logrado en el periodo 1955-1960 tasas medias anuales de incremento del producto que van desde $4.0 \%$ en España hasta $5.9 \%$ en Italia y $9.0 \%$ en Yugoslavia, y se proyectan en el período $1960-1975$ tasas de crecimiento de $5.0 \%$ al año en Portugal, $7.0 \%$ en Grecia y Turquía y $9.4 \%$ en Yugoslavia. Es evidente que en estos países la mejoría en promedio (la distribución del ingreso es también menos desigual) del nivel de vida, que se espera se efectuará a una tasa aproximadamente el doble de la hasta ahora prevista en América Latina, influirá decisivamente en el mejoramiento educativo, particularmente en las tasas de escolaridad y en la capacidad del sector público para financiar la educación media y superior. La sola velocidad del cambio económico añade un factor intangible favorable a la posi- 
bilidad de adaptar adecuadamente el sistema educativo y llevar a cabo las políticas educativas y de capacitación de la mano de obra sin conflictos de prioridad tan graves como en América Latina. Debe tenerse en cuenta que el crecimiento económico de los países del PRM y su proceso de planeación dependerán probablemente menos de la ayuda financiera externa, y estarán sujetos a menos vicisitudes de comercio exterior que en América Latina.

Hasta ahora, las tasas previsibles de crecimiento económico en América Latina se han derivado, siguiendo en esencia la metodología elaborada por la CEPAL, de la previsión de la demanda externa como factor exógeno, para estimar los incrementos necesarios de la demanda interna, a través de la industrialización y el mejoramiento agropecuario, que garanticen la meta mínima de incremento general del producto. Dicha metodología ha puesto la atención en el cambio de estructura productiva interna necesario (aunque no en los medios para lograrlo) para aumentar la oferta de bienes y, mediante una sustitución más intensa de importaciones, asegurar a la vez el equilibrio de la balanza de pagos. Pero, aparte de quedar imprecisos muchos aspectos relativos al uso de distintas tecnologías y de su efecto en los insumos de capital, los sistemas de proyecciones no se han traducido en metas de absorción de fuerza de trabajo, sean globales o sectoriales. Por supuesto que el problema metodológico es en gran parte una cuestión de falta de información adecuada, y no se desea menospreciar el gran adelanto ya logrado en la técnica de programación; se pretende solamente poner de relieve un aspecto débil de la metodología, que por fuerza tiene consecuencias prácticas muy importantes y que requerirá sin duda que se haga un esfuerzo considerable -en parte tema de la presente conferencia- encaminado a formular la estadística y los métodos de análisis indispensables. Pero, sea cual fuere el avance que se logre en metodología, puede aventurarse que lo más probable es que se llegue a la conclusión de que las metas de crecimiento actualmente consideradas en América Latina son, desde el punto de vista de la oferta de mano de obra, insuficientes.

No basta, por supuesto, un examen global de este problema. Aparte de que habrá que estudiarlo en cada país según las condiciones y los datos básicos del mismo, debe también tratarse a niveles de desagregación sectorial y subsectorial, así como geográfica (como en Italia). Puede adelantarse, a priori, que la intensa migración del ámbito rural al urbano que experimenta América Latina y que, según muchos observadores, obedece no tanto a la demanda de ocupación en la industria y los servicios, como al simple atractivo cultural de la ciudad, combinado con la repulsa que en muchas áreas ejercen las condiciones agrarias, impondrá al proceso de inđustrialización una tarea más pesada que la que normalmente se supone. El desarrollo industrial tendrá que atender a diversos criterios, no todos enteramente compatibles entre sí. Deberá absorber, no el incremento de parte de la fuerza de trabajo en general, sino muy especialmente el de la fuerza de trabajo urbana, que tiende a ser mucho más rápido. Esto supone un esfuerzo especial, difícil de lograr, para orientar el crecimiento industrial hacia tipos de actividad que - aparte de los criterios de sustitución de importaciones- supongan mayor ocupación por 
unidad de capital pero al mismo tiempo mayor producción por trabajador ocupado, a fin de contribuir a generalizar los incrementosı de la productividad y, en consecuencia, contribuir también a mejorar la distribución del ingreso.

Pero en forma simultánea, debido a la subocupación rural derivada de la baja productividad de gran parte de la agricultura latinoamericana y del hecho de que en casi todos los países la población en el campo continuará aumentando en números absolutos, la industria manufacturera estará llamada a localizarse fuera de las zonas urbanas. En varios países de América Latina se lleva ya a efecto una política de descentralización industrial, y en particular de desconcentración de las ciudades capitales, que, si bien responde a la idea de procurar mayor equilibrio regional y dar ocupación alternativa a la población rural -incluso a la que el progreso mecánico de la agricultura pueda desplazar-, pudiera estar agravando el problema de la absorción de la fuerza de trabajo en donde ésta ya existe en demasía, a niveles de calificación insuficientes, sin posibilidades de obtener educación formal, y con tendencia a aumentar. La descentralización de la industria puede también requerir ampliaciones de infraestructura -por ejemplo, en transportes y energía- que pudieran representar utilizaciones no óptimas, a la corta, de la capacidad de ahorro del país. Todo esto no quiere decir que la política de descentralización industrial no sea correcta, sino que es posible que la política de desarrollo industrial en general no haya sido planteada hasta ahora con proyección y dimensiones suficientes ante la gravedad del problema demográfico.

La absorción de mano de obra en los servicios merece, a este respecto, una consideración más cuidadosa que la que ha tenido hasta ahora en América Latina. Se tiende con frecuencia a menospreciar la ocupación en actividades de servicio, en parte por atribuirle, erróneamente, escasa o ninguna significación en la generación del producto nacional, y en parte por suponerse que es en los servicios donde existe el mayor grado de subocupación improductiva. Desde luego conviene distinguir entre "servicios" que son parte integral y necesaria del proceso de producción -como comercialización agrícola, transporte, finanzas, distribución, actividades de gobierno, mantenimiento $\mathrm{v}$ reparaciones, etc. - $\mathrm{y}$ aquellos que representan formas comercializadas del consumo de los sectores urbanos que antes se realizaban como autoconsumo -alimentación, esparcimiento, turismo, higiene y mejoramiento estético, servicios profesionales, etc.-, que a veces parecen excesivos pero que el mercado demanda. En unos y en otros pueden prevalecer formas de organización ineficientes y escalas de producción demasiado limitadas, que dan origen, sobre todo en la distribución, a que se hable de exceso de intermediarios. Parecería importante distinguir entre el efecto-ocupación del desarrollo de los servicios y la parte distributiva del ingreso que, por virtud de elementos monopólicos naturales o artificiales, se apropian actualmente. Desde el punto de vista de la ocupación, no cabe duda que la expansión de los servicios, necesariamente ligada al desarrollo industrial y al crecimiento urbano, aliviará la tarea que generalmente se asigna casi exclusivamente a la industria. El verdadero problema es evitar que 
la ocupación en los servicios sea la que en gran parte caracteriza hoy a muchas grandes ciudades latinoamericanas: los servicios de subsistencia, o sea el empleo de mano de obra no calificada en tareas de muy escasa productividad y mínimo rendimiento monetario.

IV. Lo expresado hasta aquí tiene por objeto hacer resaltar el hecho de que, no obstante los adelantos de los últimos años en materia de métodos de programación y preparación de planes nacionales de desarrollo, existen dudas e incógnitas de orden general respecto a la posibilidad de derivar de las proyecciones económicas hechas en América Latina conclusiones medianamente precisas acerca de la demanda de mano de obra y, sobre la base de ésta, indicaciones suficientes con relación al mejoramiento de los sistemas educativos y a la inversión que deba realizarse en éstos. EI método seguido en los países del PRM ha consistido, en esencia, en tomar como punto de partida las proyecciones globales y sectoriales del crecimiento económico en un período de quince años, derivar de ellas metas de producción por persona ocupada (o estimar éstas primero) y, en consecuencia, calcular el volumen de ocupación requerido (atendiendo además a otros criterios, como reducir la desocupación, absorber mayores tasas de participación por grupos de edad y sexo, etc.). El volumen de ocupación, una vez determinado por actividades, permite a su vez, según hipótesis, expresar la necesidad que habrá dentro de quince años de mano de obra por clase de ocupación, de donde se deriva el nivel y el tipo de educación; es decir, se traduce la demanda de mano de obra, en términos de calidades, en una "orden" al sistema educativo destinada a que éste "produzca" la oferta necesaria para el año-meta. Semejante orden, a la luz de la estructura y situación educativas presentes, deberá expresarse en mayores coeficientes de escolaridad, formación de maestros, ampliación de cupo en las escuelas, edificación, etc., y estos requerimientos físicos se expresan en costos de la educación, para llegar en definitiva al financiamiento de la misma.

Los informes de la OCDE sobre los países del PRM no dejan dudas sobre el carácter aproximado de los cálculos, por más que las conclusiones cualitativas sean bien claras $\mathrm{y}$, por lo demás, razonables. A falta de planes de desarrollo a largo plazo, en los países del PRM se utilizaron proyecciones de corto o mediano plazo extrapoladas o proyecciones de "perspectiva", es decir, hipótesis más o menos razonadas. En algunos casos se contó con proyecciones desagregadas en varios sectores y subsectores; en otros, las proyecciones se limitaron a los tres grandes sectores de actividad primaria, secundaria y terciaria. En cuanto a proyección de la productividad (producto por persona ocupada) se adoptó la tendencia de los diez años anteriores o se formularon, en dos casos, hipótesis que tuvieron en cuenta diversos factores y comparaciones con otros países. Mediante encuestas y tomando en cuenta informaciones sobre cambio tecnológico, datos de países más avanzados y factores especiales, se proyectaron las necesidades de empleo por clases de ocupación.

Es menos precisa la "traducción" de esta demanda en requerimientos educacionales; es decir, se adoptaron hipótesis razonadas. La dificultad consistió en gran parte en la falta de correspondencia, excep- 
to en Yugoslavia, entre las clasificaciones ocupacionales y los requisitos educativos. El problema de la clasificación ocupacional parece ser de los más graves y la derivación de los requisitos educativos, en consecuencia, el punto más delicado de las proyecciones. A esto se agrega, finalmente, la estimación del costo de la expansión y el mejoramiento del sistema educativo, y su expresión financiera.

Dadas las imprecisiones de estos cálculos (descritas en los documentos presentados por la OCDE), y el temor de que sean aún mayores en cualquier intento de aplicar la metodología al caso de los países latinoamericanos, surgen varias interrogantes. La primera se refiere a si no convendría considerar dos o más metas alternativas. No sería difícil concebir un modelo relativamente sencillo en que, por ejemplo, dada una tasa y estructura de la inversión, se consideraran diferentes crecimientos sectoriales de la producción en función de distintas hipótesis sobre incremento de la productividad, a fin de derivar, por sectores, cifras alternativas de empleo requerido. Es evidente que podrían introducirse alternativas también en las proporciones entre las clases de ocupación con sólo hacer consideraciones sobre cambios tecnológicos o cambios en la estructura de la demanda en diferentes sectores o subsectores de actividad. Aun cuando todo esto introduciría mayores complicaciones, el valor de las alternativas, sobre todo cuando se proyecta a quince años de plazo, es que da elementos de juicio que tienen importantes implicaciones para la política a seguir, lo que permitiría una mejor coordinación de la política educativa y de capacitación de mano de obra con otros aspectos de la política de desarrollo.

Una segunda interrogante proviene de lo que parece ser, en los estudios del PRM, un supuesto implícito, o sea el de que las necesidades de mano de obra a quince años de plazo se traducen en requisitos educativos referidos al sistema formal de educación exclusivamente, es decir, que se da por supuesto que la mano de obra existente y que dentro de quince años todavía forme parte de la fuerza de trabajo dispondrá, dado el nivel tecnológico y de organización industrial, de los medios de capacitación en el trabajo que pueda requerir. Tal vez en el caso de la mayor parte de los países latinoamericanos, donde no es tan evidente la posibilidad de capacitación de la mano de obra adulta, habría que ampliar el objeto del estudio para derivar de las necesidades futuras de mano de obra requisitos no sólo en materia educativa sino en cuanto a sistemas de capacitación en la industria (y en la agricultura). También, no existe actualmente en América Latina una correspondencia precisa entre el nivel y el tipo de educación, por una parte, y la clase de ocupación, por otra, por virtud, entre otras cosas, de la rapidez con que se ha generado la demanda de personal técnico en algunas actividades, el exceso de oferta en relación con la demanda en otras y, en general, por el factor de improvisación que en muchos casos prevalece. Esto quiere decir que, en la evaluación de la situación existente, habría que introducir alguna forma de corregir el nivel educativo nominal por algún factor de eficiencia en la ocupación.

En una proyección a quince años es preciso, por otra parte, con base en la evaluación del sistema educativo actual, introducir la nece- 
sidad de cambios en la técnica de la educación, particularmente en los niveles medio y superior, en los cuales se requerirán los mayores incrementos en los coeficientes de escolaridad. Dichas modificaciones en la técnica serán tanto más necesarias cuanto mayor grado de precisión se pretenda en los cálculos del costo de la expansión educativa, ya que en América Latina será inevitable que el financiamiento de la educación recaiga crecientemente en el sector público, y las presiones sobre éste provendrán también de muchos otros sectores de actividad, además de la educación (por ejemplo, de la agricultura), que tal vez no sean tan importantes en algunos países europeos. Con las técnicas educativas actuales, probablemente se llegaría a la conclusión de que no sería posible satisfacer, conforme al costo previsible, los resultados educativos de la demanda de mano de obra que se llegue a proyectar en América Latina.

V. Para poder llevar a cabo, con las salvedades indicadas, proyecciones de la demanda de mano de obra en América Latina, sería preciso establecer ciertos requisitos mínimos de información que en muchos casos no existe o que no podrá lograrse antes de los próximos censos de población en 1970. Lo primero sería determinar cuál debe ser el año-meta de las proyecciones. Parece conveniente, no obstante las deficiencias de las proyecciones económicas, adoptar como año-meta el de 1980, que será también año de censos y permitirá efectuar una serie de verificaciones y comparaciones, sobre todo si se aprovechan los censos de 1970 para introducir en ellos información y clasificaciones que permitan, a base de muestras de los mismos, obtener datos más precisos. Un período de quince años parece ser esencial, además, desde el punto de vista de la planeación del sistema educativo, en particular si se desea implantar cambios que modifiquen la calidad y no sólo la cantidad del egreso de los educandos.

Será necesario, mientras tanto, preparar elaboraciones especiales a base de los censos de 1960, mediante muestras como las tomadas en España (y recientemente en México), ${ }^{4}$ a fin de correlacionar la información sobre la actividad económica y la clase de ocupación, y ésta con el nivel y el tipo de enseñanza, por edades y por sexo. En donde sea posible, dicha información debería elaborarse no sólo al nivel nacional sino por regiones, así como por ciudades importantes. Se requerirá adoptar normas de clasificación de ocupaciones que permitan comparaciones entre los países, y, al menos con vistas a 1970 y 1980 , será conveniente establecer la correspondencia entre las ocupaciones y el nivel educativo en forma más precisa. La realización de encuestas entre empresas industriales y otras respecto a su estructura ocupacional será, como ya se ha probado en algunos de los países del PRM, de gran valor para juzgar la situación actual, $y$, si se distinguen niveles tecnológicos de las empresas, para adoptar hipótesis sobre estructura futura.

4 La de México se refiere a la del $1.5 \%$ de la población del Censo de 1960. Véase breve descripción de las tabulaciones proyectadas en: Susana Lerner, "La investigación y la planeación demográficas en México", DEMOGRAFía Y EcoNomía, Vol. I, Núm. 1, 1967, pp. 13-14. 
El estado actual de las proyecciones económicas formuladas en los países latinoamericanos no es muy satisfactorio. La mayor parte de los planes de desarrollo, algunos de los cuales han sido examinados por la Nómina de los Nueve de la OEA, se refiere a períodos relativamente cortos, de tres a cuatro años, si bien en varios casos se enmarcan en proyecciones globales y sectoriales hasta 1970 y aun 1975 . Algunos de los planes son en extremo provisionales o hipotéticos, y ha sido frecuente que se hayan efectuado revisiones sustanciales de cifras, no sólo del período histórico reciente sino de los datos del período base, lo que obliga a modificar con frecuencia las proyecciones. En realidad, se necesita hacer un esfuerzo muy considerable de mejoramiento de las proyecciones económicas en general, y de sus bases estadísticas, para poder derivar de ellas proyecciones significativas de las necesidades de mano de obra, así sea en forma muy aproximada. De cualquier manera, será conveniente que, paralelamente a las proyecciones económicas a mediano y a largo plazo, se mejoren los trabajos relativos a la información sobre mano de obra y la situación educativa, a fin de poder efectuar proyecciones provisionales de la fuerza de trabajo y de los coeficientes educativos, que a su vez serán elementos de juicio para las proyecciones generales.

Es evidente también que en la medición de la productividad existen en América Latina grandes lagunas. La importancia relativa del sector rural, y la diversidad de sus niveles tecnológicos, obligará a efectuar una desagregación no común en las estadísticas, y hasta cierto punto lo mismo hará falta en los sectores comercial e industrial. Estos son temas que podrían considerarse en la preparación de los censos económicos de 1970, pero mientras tanto tendrán que diseñarse encuestas especiales por muestreo. Indudablemente que también será preciso depurar conceptos y definiciones en los censos de 1960, para tener bases de comparabilidad. Como en el caso de los países del PRM, convendrá lograr un grado considerable de desagregación por sectores y subsectores de la clasificación uniforme de actividades industriales.

Como en todo problema relativo a la planeación en América Latina, las deficiencias estadísticas no impiden, ni deben impedir, planteamientos razonados y lógicos, ni primeras aproximaciones. En materia tan importante como el efecto-ocupación de cualquier tasa dada de crecimiento económico y de cambio estructural, no podría menos que hacerse una serie de primeros intentos con el material disponible, aprovechando la experiencia de los países que ya lo han ensayado y adaptando esa experiencia a la vez a las condiciones que se juzgue determinantes en el caso latinoamericano. 5

5 En 1966 se concluyeron, con la cooperación de la OCDE, las proyecciones de la demanda de fuerza de trabajo y de las necesidades educativas de Perú y la Argentina. Véase Instituto Nacional de Planificación y OCDE, Desarrollo económico y social, recursos humanos y educación; previsiones a largo plazo de las necesidades de mano de obra que requiere el desarrollo económico y social del Perú y sus implicaciones para el desarrollo de la educación, Lima, junio de 1966 . El estudio sobre la Argentina, hecho en cooperación con el Consejo Nacional de Desarrollo (CONADE), no ha sido dado a conocer todavía. 